\title{
Comunidade zooplanctônica e sua relação com a qualidade da água em reservatórios do Estado de São Paulo
}

\author{
Bruno P. De-Carli ${ }^{1} 2$ (1) https://orcid.org/0000-0002-0836-1244 \\ Felícia P. de Albuquerque' (1D https://orcid.org/0000-0002-481 1-5195 \\ Viviane Moschini-Carlos' (1) https://orcid.org/0000-0002-5832-912X \\ Marcelo Pompêo 3 (i) https://orcid.org/0000-0002-5632-9257
}
1. Programa de Pós-Graduação em Ciências Ambientais do Instituto de Ciência e Tecnologia, UNESP Sorocaba, Av. Três de Março, 51 1, 18087-180 Sorocaba,SP, Brasil. (felicia.pa@hotmail.com, viviane@sorocaba.unesp.br)
2. Universidade Paulista, Campus Rangel, Av. Francisco Manoel, s/n, 1 1045-300 Santos, SP, Brasil. (bpdecarli@yahoo.com.br)
3. Departamento de Ecologia, Instituto de Biociências, Universidade de São Paulo (USP), Rua do Matão, 321, Trav. 14, Cidade Universitária, 05508-090 São Paulo, SP, Brasil.

Recebido 26 Outubro 2017

Aceito 19 Março 2018

Publicado 11 Junho 2018

DOI 10.1590/1678-4766e2018013

\begin{abstract}
Zooplankton community and their relationship with water quality in São Paulo state reservoirs. Zooplanktonic communities are important components in trophic chain considering primary producers and other levels. In addition, some species are sensitive or tolerant to organic pollution. Here we test the hypothesis that species composition varies according to the eutrophication gradient. We sought to establish which environmental factors are responsible for the variation of the species distribution. This study aimed to characterize zooplankton community and to establish a link between water quality in São Paulo State reservoirs. Samples were collected through vertical hauls with plankton net $(68 \mu \mathrm{m})$ in seven water bodies. All specimens were identified with specific literature and counted in Sedgwick rafter chamber. Abiotic and biotic data were analyzed by a canonical correspondence analysis. A total of 67 taxa was identified, being Rotifera predominant. The reservoirs exhibited eutrophic conditions mainly Salto Grande, Barra Bonita and Broa. The results point out the rotifers Trichocerca longiseta (Schrank, 1802), Hexarthra sp., Brachiounus spp. and Keratella tropica (Apstein, 1907) followed eutrophication gradient. Additionally, microcrustaceans such as Notodiaptomus, Mesocyclops, Metacyclops and Diaphanossoma occurred in eutrophic reservoirs. On other hand, the cladocerans Ceriodaphnia cornuta (Sars, 1886) and Bosminopsis deitersi Richard, 1895 were considered indicators of better water quality due to relationship with transparency. The results point out that some indicator species are effective in environmental monitoring without reject physico-chemical and biological analysis.
\end{abstract}

KEYWORDS. Zooplankton, eutrophication, water quality.

RESUMO. As comunidades zooplanctônicas são importantes componentes na cadeia alimentar considerando os produtores primários e os demais níveis. Além disso, algumas espécies são sensíveis ou tolerantes à poluição orgânica. Aqui testamos a hipótese de que a composição de espécies varia conforme o gradiente de eutrofização. Buscou-se estabelecer os possíveis fatores ambientais responsáveis pela variação na distribuição das espécies. Esse estudo teve como objetivo caracterizar a comunidade zooplanctônica e relacionar os dados com a qualidade da água em reservatórios do Estado de São Paulo. Amostras foram coletadas através de arrasto vertical por meio de rede de plâncton $(68 \mu \mathrm{m})$ em sete corpos d'água. Todos os espécimes foram identificados através de literatura específica e contados em camâra de Sedgwick rafter. Os dados bióticos e abióticos foram analisados através da análise de correspondência canônica. Um total de 67 táxons foram encontrados, sendo Rotifera o predominante. No geral, os reservatórios apresentaram condições eutróficas, principalmente Salto Grande, Barra Bonita e Broa. Os resultados apontaram que os rotíferos Trichocerca longiseta (Schrank, 1802), Hexarthra sp., Brachiounus spp. e Keratella tropica (Apstein, 1907) seguiram o gradiente de eutrofização. Além desses, microcrustáceos como Notodiaptomus, Mesocyclops, Metacyclops e Diaphanossoma ocorreram em reservatórios eutróficos. Por outro lado, os cládoceros Ceriodaphnia cornuta (Sars, 1886) e Bosminopsis deitersi Richard, 1895 foram considerados indicadores de melhor qualidade devido à relação com águas mais transparentes. Os resultados apontam que algumas espécies do zooplâncton são efetivas para o monitoramento ambiental sem descartar as análises físico-químicas e biológicas.

PALAVRAS-CHAVE. Zooplâncton, eutrofização, qualidade da água.

A disponibilidade dos recursos hídricos com qualidade adequada tem um papel fundamental no desenvolvimento socioeconômico do Brasil. Diante disso, análises estratégicas da atual situação e perspectivas para o futuro das águas no país são fundamentais, afim de estabelecer políticas públicas e condições para o desenvolvimento sustentável (TUNDISI, 2014).
As principais fontes de poluição aquática são os resíduos domiciliares, industriais e agrícolas (TuNDISI, 2008; CAMPos et al., 2014). A contaminação por esgoto doméstico é observada principalmente nos países onde a infraestrutura de saneamento é deficitária (TuNDISI, 1994). O despejo in natura pode desencadear a eutrofização e consequentemente o aumento de cianobactérias, resultando em um quadro 
conhecido na região metropolitana de São Paulo, onde verifica-se uma piora na qualidade da água, contaminação por poluentes emergentes e uso intenso de algicidas (LoPEZDoval et al., 2017).

Nos ambientes aquáticos, a comunidade zooplanctônica é constituída de animais invertebrados microscópicos que vivem em suspensão, incluindo principalmente rotíferos, cladóceros e copépodes (TUNDIsI \& MATSUMURA-TundisI, 2008). Além destes, podem ser encontradas larvas de insetos, moluscos, nematóides, ostracodes, entre outros (CETESB, 2012; De-Carli et al., 2017a). Devido ao curto ciclo de vida e à sensibilidade de algumas espécies às mudanças ambientais, os organismos zooplanctônicos são considerados bioindicadores nos ecossistemas aquáticos (SANTOS et al., 2009). Esses organismos também são considerados um elo entre os produtores primários e demais níveis tróficos, além de promover a ciclagem de nutrientes e a manutenção das cadeias tróficas (BARBosa et al., 2006; KUHLMANN et al., 2012).

Pesquisas sobre o zooplâncton são realizadas desde a década de 1970 no Brasil (MATSUMURA-TUNDISI \& TUNDISI, 1976; Nordi \& Watanabe, 1978; Matsumura-TundisI et al., 1989). Destacam-se os trabalhos sobre qualidade ambiental e eutrofização (MATSUMURA-TUNDISI \& TUNDISI, 2005; SENDACZ et al., 2006; Sousa et al. 2008; LoureIro et al., 2011; ZAGANINI et al., 2011; SouZA et al., 2013, Gazonato-Neto et al., 2014; AraúJo \& NogueIra, 2016). A proposta desse trabalho foi verificar se há diferenças na composição do zooplâncton de acordo com o gradiente de eutrofização e quais fatores ambientais são responsáveis pela variação da distribuição das espécies. Portanto, esse estudo teve como objetivo caracterizar a comunidade zooplanctônica e relacionador os dados com a qualidade da água em sete reservatórios localizados no Estado de São Paulo.

\section{MATERIAL E MÉTODOS}

As amostragens foram realizadas nos reservatórios de Barra Bonita (06/2015), Rio Grande (08/2015), Broa (06/2015), Salto Grande (06/2015), Atibainha (10/2015), Itupararanga (09/2015) e Igaratá (09/2015), totalizando 21 pontos de amostragem. O critério de escolha dos reservatórios foi baseado no conceito de tipologia preconizado pela Diretiva Quadro da Água (Ec, 2012). Em cada reservatório foram coletadas amostras em três zonas distintas (KIMMEL et al., 1990): uma na porção rio localizada na parte alta do reservatório, na zona intermediária e próximo da barragem (Fig. 1). Nesses locais, a transparência (m) foi mensurada através do desaparecimento do disco de Secchi (DS) e a profundidade máxima através de sonda. Para a determinação da zona fótica multiplicou-se o valor do DS por 2,7 (COLE, 1979). O oxigênio dissolvido (mg/L), a condutividade elétrica $(\mu \mathrm{S} / \mathrm{cm}), \mathrm{pH}$ e temperatura $\left({ }^{\circ} \mathrm{C}\right)$ foram medidos através de sonda multiparâmetros (YSI mod 556 MPS). Com a utilização de mangueiras (NAVARro et al., 2006; PÁDUA, 2009; BeCKER et al., 2010), amostras integradas foram obtidas na zona fótica para determinação dos sólidos em suspensão $(\mathrm{mg} / \mathrm{L})$, clorofila-a $(\mu \mathrm{g} / \mathrm{L})$ e nutrientes $(\mu \mathrm{g} / \mathrm{L})$ (nitrato, nitrito, amônio, nitrogênio inorgânico dissolvido e fósforo total) (Tab. I). Os níveis de trofia foram calculados por meio do índice de estado trófico para reservatórios (CUNHA et al., 2013).

$\mathrm{O}$ zooplâncton foi coletado por meio de arrasto vertical com rede de $68 \mu \mathrm{m}$ na extensão da profundidade da zona fótica. $\mathrm{O}$ volume filtrado foi estimado pela medida de profundidade do arrasto e do raio da boca da rede (CETESB, 2012). Os indivíduos foram identificados através de Koste (1978), Elmoor-Loureiro (1997), Nogrady \& SEgers (2002), Silva (2008) e Perbiche-Neves et al. (2015). Para a quantificação dos organismos foi utilizada uma câmara de Sedgwick-Rafter com capacidade de $1 \mathrm{ml}$ retirandose alíquotas da amostra total homogeneizada contando no mínimo 100 indivíduos do táxon mais abundante (CETESB, 2012; FreitAs et al., 2012). A riqueza foi representada pelo número de espécies e a densidade numérica expressa em organismos por metro cúbico $\left(\right.$ org. $\left./ \mathrm{m}^{3}\right)$. A classificação da frequência de ocorrência foi baseada em DAJOz (1983) e CASTILHO et al. (2016): espécies constantes ocorrem em mais de $50 \%$ das amostras, de $25 \%$ a $50 \%$ são consideradas espécies acessórias e percentuais menores que $25 \%$ espécies acidentais.

Com intuito de verificar diferenças na riqueza e abundância totais do zooplâncton considerando o gradiente de trofia, aplicou-se uma análise de variância (ANOVA) ao nível de significância de $5 \%$. Somente após a verificação dos pressupostos através dos testes de Shapiro Wilk (normalidade) e Levene (homocestadicidade), a ANOVA foi aplicada (ZAR, 2010). Para verificar a associação entre o zooplâncton e as variáveis limnológicas, foi aplicada a análise de correspondência canônica (ACC) com auxílio do software Canoco 4.5 (Legendre \& LEGENDRE, 1998). Utilizou-se duas matrizes de dados previamente padronizados através de transformação logarítimica $(\log \mathrm{x}+1)$ e ranging (valor bruto-valor mínimo/valor máximo-valor mínimo) (Milligan \& COOPER, 1988) sendo: ambientais (nitrogênio inorgânico dissolvido, fósforo total, clorofila-a, transparência, sólidos em suspensão, temperatura, condutividade elétrica, pH e oxigênio dissolvido) e abundância de espécies. Aplicouse o teste de Monte Carlo (999 permutações) para avaliar a significância da ACC (VAN DEN BRINK \& TER BRAAK, 1998). As amostras do zooplâncton estão depositadas no Laboratório de Limnologia do Departamento de Ecologia da Universidade de São Paulo (USP, São Paulo, SP).

\section{RESULTADOS}

Maiores profundidades e níveis de transparência foram observadas no reservatório Igaratá. Para a condutividade elétrica e nutrientes, os maiores valores foram registrados nas represas Salto Grande e Barra Bonita. Em relação a clorofila-a, foi observado teores mais elevados na represa do Broa. A maioria das estações de coleta foi classificada como eutrófica, exceto para alguns locais dos reservatórios Atibainha e Igaratá, considerados mesotróficos e oligotróficos (Tab. II). 

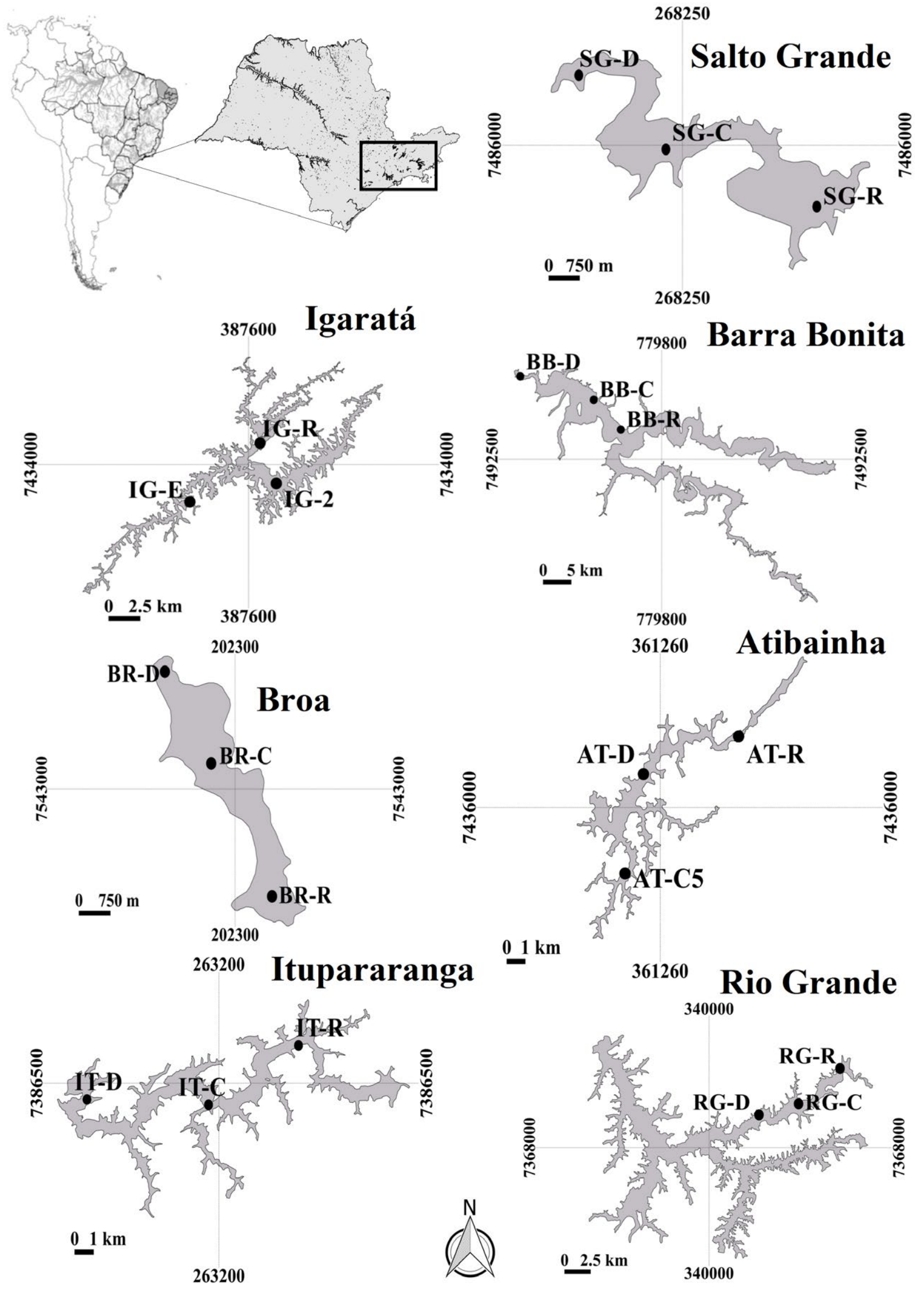

Fig. 1. Mapa dos pontos de amostragem nos reservatórios Salto Grande (SG), Barra Bonita (BB), Broa (BR), Itupararanga (IT), Atibainha (AT), Rio Grande (RG) e Igaratá (IG), Estado de São Paulo, Brasil em 2015 (R e 2, entrada de água; C, região central; D, barragem; C5, canal; E, saída de água). 
Tab. I. Variáveis limnológicas e métodos empregados nos reservatórios Salto Grande, Barra Bonita, Broa, Itupararanga, Atibainha, Rio Grande e Igaratá, Estado de São Paulo, Brasil em 2015.

\begin{tabular}{lll}
\hline Variáveis & Métodos & Referências \\
\hline oxigênio dissolvido & Sonda & - \\
temperatura & Sonda & - \\
condutividade elétrica & Sonda & - \\
$\mathrm{pH}$ & Sonda & - \\
transparência & Disco de Secchi & - \\
material em suspensão & Gravimétrico & TeIXEIRA et al. (1965) \\
fósforo total & Espectrofotométrico & VALDERRAMA (1981) \\
nitrato & Espectrofotométrico & MACKERETH et al. $(1978)$ \\
nitrito & Espectrofotométrico & MACKERETH et al. (1978) \\
amônio & Espectrofotométrico & KOROLEFF $(1976)$ \\
nitrogênio inorgânico dissolvido & $\sum \mathrm{NO}_{2}^{-}+\mathrm{NO}_{3}^{-}+\mathrm{NH}_{4}^{+}$ & WETZEL \& LiKENS (1991) \\
clorofila-a & Espectrofotométrico & LORENZEN (1967) \\
\hline
\end{tabular}

Tab. II. Variáveis limnológicas obtidas nos reservatórios Salto Grande (SG), Barra Bonita (BB), Broa (BR), Itupararanga (IT), Atibainha (AT), Rio Grande (RG) e Igaratá (IG), Estado de São Paulo, Brasil em 2015 (R e 2, entrada de água; C, região central; D, barragem; C5, canal; E, saída de água; PROF, profundidade máxima; T, temperatura; DO, oxigênio dissolvido; $\mathrm{pH}$, potencial hidrogeniônico; EC, condutividade elétrica; DS, transparência; SS, sólidos em suspensão; DIN, nitrogênio inorgânico dissolvido; TP, fósforo total; CLa, clorofila-a; TSI, índice de estado trófico; HI, hipereutrófico; $\mathrm{SU}$, supertrófico; EU, eutrófico; ME, mesotrófico; OL, oligotrófico).

\begin{tabular}{|c|c|c|c|c|c|c|c|c|c|c|c|c|}
\hline Estação & $\begin{array}{c}\text { PROF } \\
\mathrm{m}\end{array}$ & $\begin{array}{c}\mathrm{T} \\
{ }^{\circ} \mathrm{C} \\
\end{array}$ & $\begin{array}{c}\mathrm{DO} \\
\mathrm{mg} / \mathrm{L}\end{array}$ & $\mathrm{pH}$ & $\begin{array}{c}\mathrm{EC} \\
\mu \mathrm{S} / \mathrm{cm}\end{array}$ & $\begin{array}{c}\mathrm{DS} \\
\mathrm{m}\end{array}$ & $\begin{array}{c}\mathrm{SS} \\
\mathrm{mg} / \mathrm{L}\end{array}$ & $\begin{array}{l}\mathrm{DIN} \\
\mu \mathrm{g} / \mathrm{L}\end{array}$ & $\begin{array}{c}\mathrm{TP} \\
\mu \mathrm{g} / \mathrm{L} \\
\end{array}$ & $\begin{array}{l}\text { CLa } \\
\mu \mathrm{g} / \mathrm{L}\end{array}$ & \multicolumn{2}{|c|}{ TSI } \\
\hline SG-R & 5,5 & 20,87 & 3,22 & 6,78 & 410 & 1,24 & 8,00 & 908,32 & 115,30 & 26,73 & 69 & $\mathrm{HI}$ \\
\hline SG-C & 8,4 & 20,92 & 3,16 & 7,12 & 363 & 1,07 & 8,14 & 355,78 & 100,19 & 13,75 & 67 & SU \\
\hline SG-D & 11,0 & 21,25 & 2,71 & 6,86 & 340 & 1,19 & 7,71 & 399,25 & 69,12 & 19,38 & 67 & $\mathrm{SU}$ \\
\hline BB-R & 16,4 & 22,23 & 4,01 & 7,01 & 274 & 1,55 & 3,90 & 2063,54 & 95,15 & 15,64 & 67 & SU \\
\hline BB-C & 18,3 & 20,99 & 3,56 & 6,80 & 258 & 2,50 & 3,10 & 845,29 & 64,93 & 11,63 & 65 & SU \\
\hline BB-D & 22,6 & 22,41 & 2,80 & 6,88 & 247 & 2,90 & 3,00 & 382,94 & 70,80 & 3,74 & 63 & $\mathrm{EU}$ \\
\hline BR-R & 3,2 & 22,57 & 5,69 & 7,11 & 18 & 0,63 & 7,75 & 66,90 & 28,83 & 30,07 & 65 & SU \\
\hline BR-C & 6,4 & 22,57 & 4,26 & 7,46 & 17 & 0,62 & 6,75 & 27,75 & 18,75 & 29,74 & 64 & SU \\
\hline BR-D & 13,0 & 21,56 & 4,11 & 7,90 & 17 & 0,62 & 10,33 & 38,01 & 27,99 & 38,09 & 66 & $\mathrm{SU}$ \\
\hline IT-R & 8,2 & 17,91 & 4,40 & 7,28 & 83 & 1,23 & 6,40 & 89,25 & 28,83 & 8,82 & 62 & $\mathrm{EU}$ \\
\hline IT-C & 10,8 & 19,38 & 2,97 & 6,59 & 81 & 1,61 & 2,80 & 60,66 & 13,71 & 5,35 & 59 & $\mathrm{ME}$ \\
\hline IT-D & 14,8 & 19,25 & 3,65 & 6,81 & 80 & 1,39 & 3,60 & 63,67 & 14,55 & 9,76 & 60 & $\mathrm{EU}$ \\
\hline AT-R & 8,9 & 24,96 & 4,08 & 7,51 & 41 & 1,88 & 2,57 & 244,32 & 7,50 & 7,64 & 58 & $\mathrm{ME}$ \\
\hline AT-C & 19,1 & 24,33 & 3,52 & 7,85 & 40 & 2,65 & 2,12 & 102,67 & 7,50 & 5,51 & 57 & $\mathrm{ME}$ \\
\hline AT-C5 & 17,0 & 24,17 & 2,57 & 7,98 & 39 & 2,38 & 2,25 & 73,74 & 7,50 & 2,17 & 55 & $\mathrm{ME}$ \\
\hline RG-R & 4,4 & 22,59 & 2,61 & 2,61 & 108 & 1,12 & 4,17 & 571,39 & 33,86 & 5,16 & 61 & $\mathrm{EU}$ \\
\hline RG-C & 7,5 & 22,57 & 3,20 & 3,20 & 104 & 1,52 & 0,50 & 604,83 & 21,27 & 5,12 & 60 & $\mathrm{EU}$ \\
\hline RG-D & 11,7 & 22,61 & 5,15 & 5,15 & 105 & 1,71 & 6,83 & 663,52 & 7,50 & 9,80 & 58 & $\mathrm{ME}$ \\
\hline IG-R & 28,6 & 22,57 & 4,94 & 5,76 & 33 & 5,15 & 0,60 & 106,40 & 7,50 & 0,40 & 50 & OL \\
\hline IG-2 & 44,0 & 22,66 & 3,51 & 3,74 & 35 & 3,95 & 0,90 & 104,73 & 17,07 & 0,67 & 54 & $\mathrm{ME}$ \\
\hline IG-E & 24,5 & 23,44 & 3,18 & 6,80 & 36 & 2,92 & 2,00 & 73,50 & 7,50 & 3,61 & 56 & $\mathrm{ME}$ \\
\hline
\end{tabular}

Sessenta e sete táxons zooplanctônicos foram identificados, compostos por organismos das ordens Calanoida e Cyclopoida (ambos Copepoda, Crustacea), ordem Cladocera (Crustacea), Filo Rotifera, Filo Protozoa, ordem Diptera (Insecta) e Filo Nematoda (Tab. III). Rotifera apresentou maior riqueza (33 táxons) e, incluída nesse grupo, Brachionidae foi considerada a família mais representativa (14 táxons). Em relação aos microcrustáceos, o segundo em termos de riqueza (29 táxons), Cyclopidae foi considerada mais representativa (7 táxons). No que se refere aos pontos de amostragem, o maior número de espécies foi registrado na parte central do reservatório Salto Grande, em contraste com a região de barragem no reservatório Rio Grande (Fig. 2). No entanto, considerando os dados de riqueza total agrupados segundo os graus de trofia (hipereutrófico, eutrófico e mesotrófico), constatou-se que as médias não diferiram estatisticamente $(\mathrm{F}=2,164 ; \mathrm{p}=0,1473)$.

Os táxons considerados constantes $(>50 \%)$ foram nauplius de Calanoida, nauplius e copepoditos de Cyclopoida, Thermocyclops decipens (Kiefer, 1929), Thermocyclops minutus (Lowndes, 1934), Bosmina sp., Asplanchna sp., Collotheca sp., Kellicottia bostoniensis (Rousselet, 1908), Keratella cochlearis (Gosse,1851) e Difflugia sp. Os rotíferos Asplanchna sp. e K. bostoniensis, além dos nauplius e copepoditos de Cyclopoida, foram mais abundantes considerando os sete reservatórios. Maiores abundâncias foram verificadas na represa de Itupararanga e as menores no reservatório do Broa (Fig.3). Ao agrupar a abundância total das espécies conforme os graus de trofia (hipereutrófico, eutrófico e mesotrófico), verificou-se que as médias não apresentaram diferenças significativas $(\mathrm{F}=1,618 ; \mathrm{p}=0,2291)$. 
Tab. III. Lista e frequência relativa (FR) dos táxons nos reservatórios Salto Grande, Barra Bonita, Broa, Itupararanga, Atibainha, Rio Grande e Igaratá, Estado de São Paulo, Brasil em 2015 (NI, não identificado).

\begin{tabular}{|c|c|c|c|}
\hline CALANOIDA & FR $(\%)$ & ROTIFERA (cont.) & FR (\%) \\
\hline Copepodito & 41 & Asplanchna sp. & 52 \\
\hline Nauplius & 67 & Brachionus angularis Gosse, 1851 & 30 \\
\hline Notodiaptomus cearensis (Wright, 1936) & 19 & Brachionus caudatus Barrois \& Daday, 1894 & 41 \\
\hline Notodiaptomus conifer (Sars, 1901) & 4 & Brachionus falcatus Zacharias, 1898 & 4 \\
\hline Notodiaptomus henseni (Dahl, 1894) & 37 & Brachionus mirus Daday, 1905 & 4 \\
\hline Notodiaptomus iheringi (Wright, 1935) & 11 & Brachionus plicatilis Müller, 1786 & 7 \\
\hline Notodiaptomus sp. & 44 & Brachionus sp. & 11 \\
\hline N.I & 7 & Brachionus variabilis Hempel, 1896 & 4 \\
\hline CYCLOPOIDA & & Collotheca sp. & 67 \\
\hline Copepodito & 74 & Conochilus unicornis Rousselet, 1892 & 26 \\
\hline Nauplius & 74 & Epiphanes sp. & 4 \\
\hline Mesocyclops longisetus (Thiebaud, 1912) & 19 & Euchlanis sp. & 11 \\
\hline Mesocyclops ogunnus Onabamiro, 1957 & 4 & Filinia opoliensis (Zacharias, 1898) & 11 \\
\hline Mesocyclops sp. & 44 & Filinia sp. & 15 \\
\hline Metacyclops sp. & 4 & Hexarthra sp. & 4 \\
\hline Thermocyclops decipiens (Kiefer, 1929) & 52 & Kellicottia bostoniensis (Rousselet,1908) & 74 \\
\hline Thermocyclops minutus (Lowndes, 1934) & 52 & Keratella americana Carlin, 1943 & 41 \\
\hline Thermocyclops sp. & 4 & Keratella cochlearis (Gosse,1851) & 59 \\
\hline CLADOCERA & & Keratella quadrata (Müller, 1786) & 7 \\
\hline Alona sp. & 4 & Keratella sp. & 30 \\
\hline Bosmina freyi De Melo \& Hebert, 1994 & 11 & Keratella tropica (Apstein, 1907) & 22 \\
\hline Bosmina sp. & 63 & Lecane sp. & 11 \\
\hline Bosminopsis deitersi Richard, 1895 & 19 & Lepadella sp. & 4 \\
\hline Ceriodaphnia cornuta (Sars, 1885) & 26 & Polyarthra vulgaris Carlin, 1943 & 41 \\
\hline Ceriodaphnia silvestrii Daday, 1902 & 7 & Pompholyx sp. & 15 \\
\hline Ceriodaphnia sp. & 41 & Ptygura sp. & 22 \\
\hline Chydoridae & 4 & Synchaeta stylata Wierzejski, 1893 & 19 \\
\hline Daphnia gessneri (Herbst, 1967) & 26 & Trichocerca capucina (Wierzejski \& Zacharias, 1893) & 4 \\
\hline Daphnia sp. & 4 & Trichocerca longiseta (Schrank, 1802) & 4 \\
\hline Diaphanosoma sp. & 44 & Trichocerca sp. Lamarck, 1801 & 41 \\
\hline Diaphanosoma spinulosum Herbst, 1975 & 19 & PROTOZOA & \\
\hline Moina sp. & 19 & Difflugia sp. & 52 \\
\hline ROTIFERA & & N.I. & 19 \\
\hline Anuraeopsis sp. & 7 & DIPTERA & \\
\hline Ascomorpha sp. & 33 & Chaoborus sp. & 11 \\
\hline \multirow[t]{2}{*}{ Asplanchna brightwellii Gosse, 1850} & 11 & NEMATODA & \\
\hline & & N.I. & 4 \\
\hline
\end{tabular}

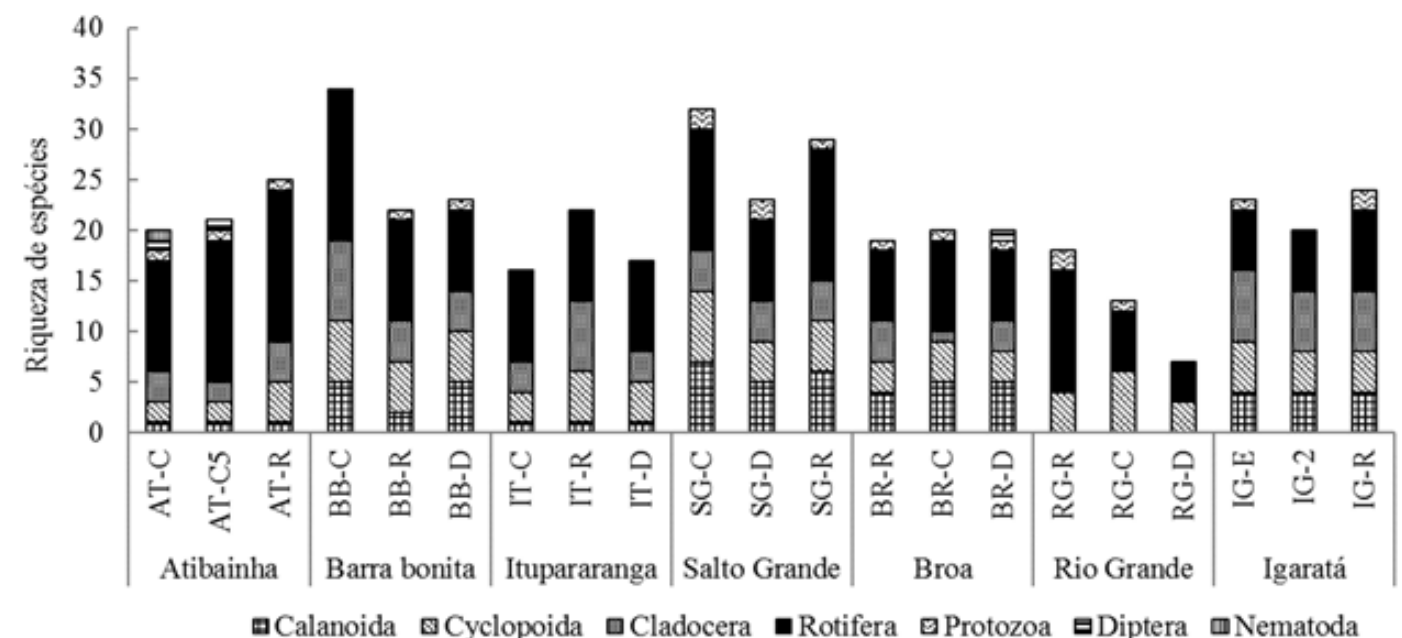

Fig. 2. Riqueza taxonômica da comunidade zooplanctônica nos reservatórios Salto Grande, Barra Bonita, Broa, Itupararanga, Atibainha, Rio Grande e Igaratá, Estado de São Paulo, Brasil em 2015. 


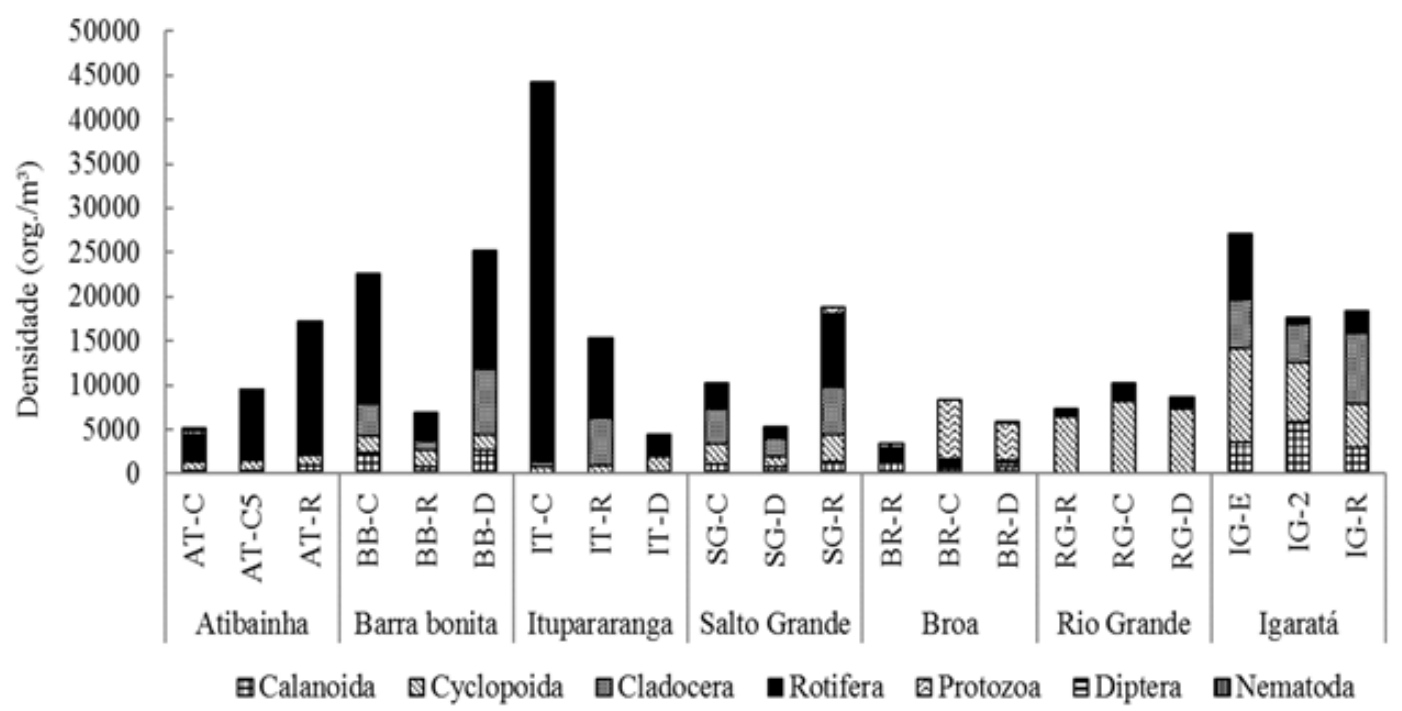

Fig. 3. Densidade da comunidade zooplanctônica nos reservatórios Salto Grande, Barra Bonita, Broa, Itupararanga, Atibainha, Rio Grande e Igaratá, Estado de São Paulo, Brasil em 2015.

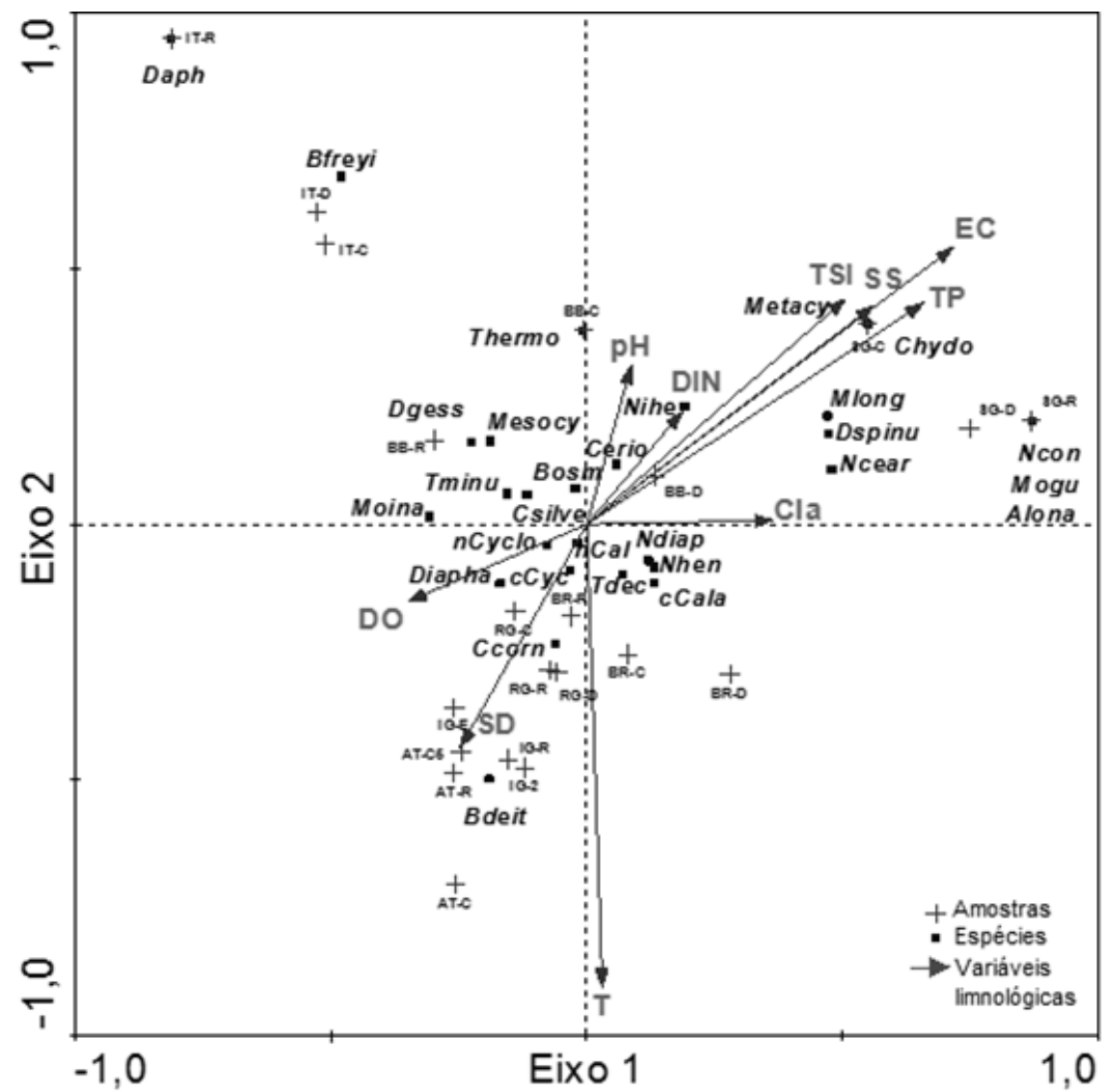

Fig. 4. Gráfico da análise de correspondência canônica relacionandos a abundância dos microcrustáceos com as variáveis limnológicas nos reservatórios Salto Grande (SG), Barra Bonita (BB), Broa (BR), Itupararanga (IT), Atibainha (AT), Rio Grande (RG) e Igaratá (IG), Estado de São Paulo, Brasil em 2015 (R e 2, entrada de água; C, região central; D, barragem; C5, canal; OD, oxigênio dissolvido; SD, transparência da água; potencial hidrogeniônico $(\mathrm{pH})$; nitrogênio inorgânico dissolvido (DIN); estado trófico (TSI); sólidos em suspensão (SS); fósforo total (TP); clorofila-a (Cla); condutividade (EC); Daphnia sp. (Daph); Bosmina freyi (Bfreyi); Thermocyclops sp. (Thermo); Daphnia guessneri (Dgess); Mesocyclops sp. (Mesocy); Bosmina sp. (Bosm); Moina sp. (Moina); Thermocyclops minutus (Tmin); Ceriodaphnia silvestrii (Csilve); Ceriopdaphnia sp. (Cerio); Notodiaptomus iheringi (Nihe); Metacyclops sp. (Meta); Chydoridae (Chydo); Mesocyclops longisetus (Mlong); Diaphanosoma spinulosum (Dspinu); Notodiaptomus cearensis (Ncear); Notodiaptomus conifer (Ncon); Mesocyclops ogunnus (Mogu); Alona sp. (Alona); nauplius Cyclopoida (nCyclo); copepodito Cyclopoida (cCyc); Ceriodaphnia cornuta (Ccorn); nauplius Calanoida (nCal); Bosminopsis deitersi (Bdeit); Notodiaptomus sp. (Ndiap); Thermocyclops decipens (Tdec); Notodiaptomus henseni (Nhen); copepodito Calanoida (cCala). 


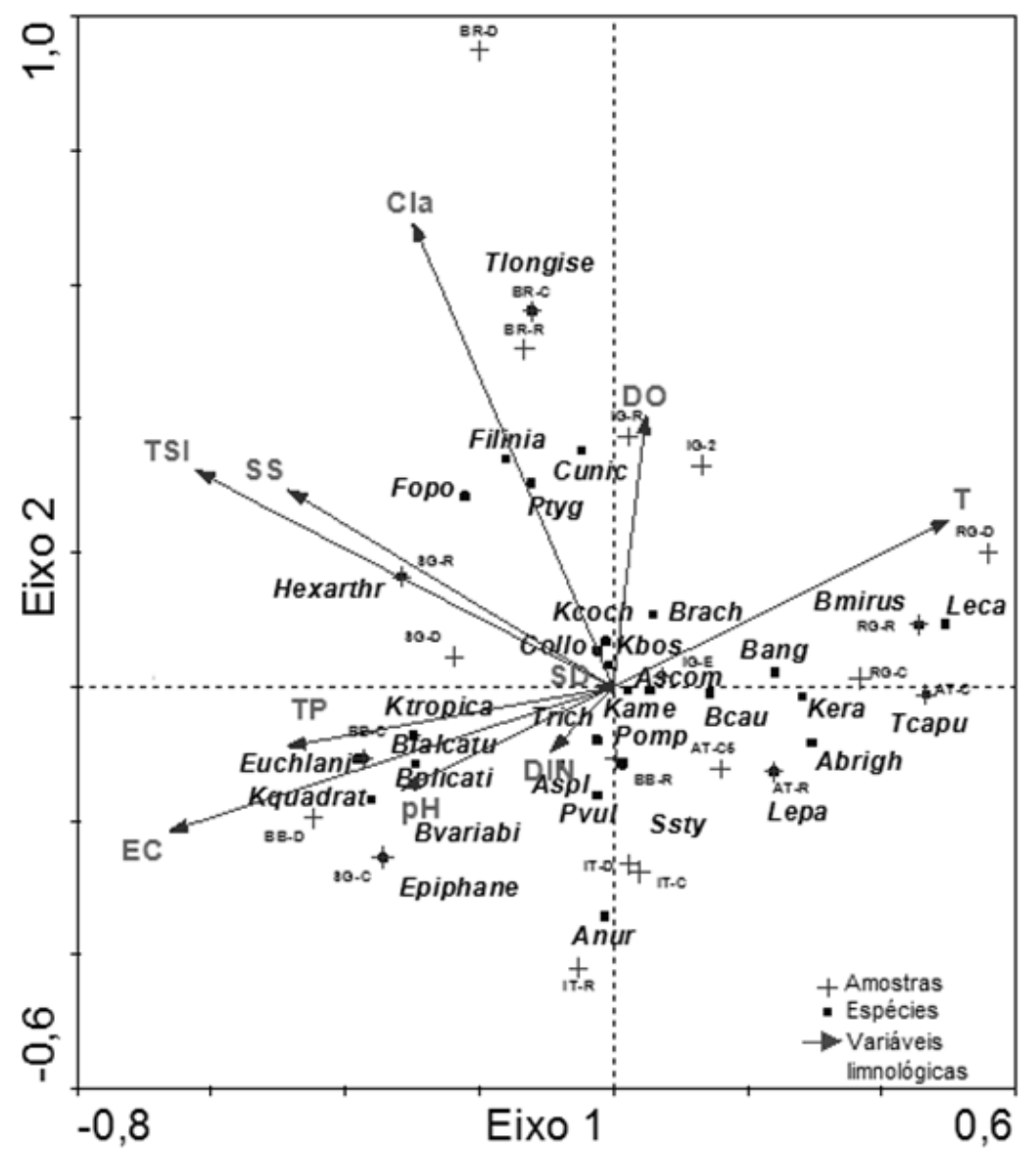

Fig. 5. Gráfico da análise de correspondência canônica relacionando a abundância dos rotíferos com as variáveis limnológicas nos reservatórios Salto Grande (SG), Barra Bonita (BB), Broa (BR), Itupararanga (IT), Atibainha (AT), Rio Grande (RG) e Igaratá (IG), Estado de São Paulo, Brasil em 2015 (R e 2, entrada de água; $\mathrm{C}$, região central; D, barragem; C5, canal; oxigênio dissolvido (OD); transparência da água (SD); potencial hidrogeniônico $(\mathrm{pH})$; nitrogênio inorgânico dissolvido (DIN); estado trófico (TSI); sólidos em suspensão (SS); fósforo total (TP); clorofila-a (Cla); condutividade (EC); Trichocerca longiseta (Tlongise); Filinia sp. (Filinia); Filinia opoliensis (Fopo); Hexarthra sp. (Hexarthr); Ptygura sp. (Ptyg); Keratella cochlearis (Kcoch); Collotheca sp. (Collo); Conochilus unicornis (Cunic); Trichocerca (Tricho); Keratella tropica (Ktropica); Brachionus falcatus (Bfalcatu); Brachionus plicatilis (Bplicati); Keratella quadrata (Kquadrat); Euchlanis sp. (Euchlani); Brachionus variabilis (Bvariabi); Asplanchna sp. (Aspl); Polyarthra vulgaris (Pvul); Anuraeopsis sp. (Anur); Brachionus sp. (Brach); Kellicottia bostoniensis (Kbosto); Ascomorpha sp. (Asco); Keratella americana (Kame); Brachionus mirus (Bmirus); Pompholyx sp. (Pomp); Brachionus angularis (Bang); Brachionus caudatus (Bcau); Synchaeta stylata (Ssty); Lepadella sp. (Lepa); Keratella sp. (Kera); Asplanchna brightwellii (Abrigh); Trichocerca capucina (Tcapu).

Com relação a ACC (Figs 4, 5), no grupo dos rotíferos e crustáceos foram observadas correlações acima de 0,90 na relação espécies-ambiente $(p<0,01)$. Para os microcrustáceos, os dois primeiros eixos explicaram 50\% de variância total. Nos rotíferos, os dois primeiros eixos explicaram 39\% da variação observada. Com os demais grupos (Diptera, Nematoda e Protozoa) não foi verificado resultado significante para a ACC $(\mathrm{p}>0,01)$.

Na represa Salto Grande, a abundância dos crustáceos Metacyclops sp., Chydoridae, Mesocyclops longisetus, Diaphanosoma spinulosum, Notodiaptomus cearensis, Notodiaptomus conifer, Mesocyclops ogunnus e Alona sp. esteve relacionada com o estado trófico, sólidos em suspensão, fósforo e condutividade. Nos reservatórios Rio Grande, Igaratá e Atibainha, os cladóceros Diaphanossoma sp. e Bosminopsis deitersi foram registrados em locais com maior transparência.
No reservatório Rio Grande, a temperatura influenciou as populações de Brachionus mirus e Lecane sp. Filinia opoliensis, Filinia sp., Conochilus unicornis, Ptygura sp. e Trichocerca longiseta estiveram associados com a clorofila-a. No reservatório Salto Grande, o rotífero Hexarthra sp. esteve relacionado com o estado trófico e sólidos em suspensão. As concentrações de fósforo total, condutividade e $\mathrm{pH}$ nas densidades dos táxons Brachionus spp., Euchlanis sp., Keratella tropica e Keratella quadrata na represa Barra Bonita.

\section{DISCUSSÃO}

Com base nos parâmetros limnológicos, foi possível observar locais que variam de oligotróficos a hipereutróficos, sendo a maioria eutrófico $(61,9 \%)$. Além disso, baixos níveis de oxigênio dissolvido também foram mensurados. Tanto 
a variação de trofia como os baixos níveis de oxigênio dissolvido podem implicar em alterações na dinâmica populacional do zooplâncton (ZAGANINI et al., 2011; DANTASSiLVA \& DANTAS, 2013; VAD et al., 2013). No entanto, não foi possível detectar diferenças significativas na riqueza e abundância do zooplâncton entre os diferentes estados tróficos. Esse fato corrobora com a hipótese rejeitada por ZAGANINI et al. (2011), de que o zooplâncton suporta o forte grau de impacto da poluição, assemelhando-se qualiquantitativamente em diferentes condições ambientais.

No presente estudo, assim como em outros reservatórios, os rotíferos atingiram maior riqueza (Sendacz et al., 1985; Almeida et al., 2006; Meirinho \& POMPÊo, 2015); são organismos oportunistas e podem se adaptar às diferentes condições ambientais assim como causar desequilíbrio na estrutura da comunidade zooplanctônica devido à competição por recursos alimentares. Esse grupo têm capacidade de ingerir pequenas partículas, bactérias e detritos orgânicos que são abundantes em sistemas mais produtivos (AllaN, 1976; LouREIRo et al., 2011).

Observou-se que Brachionus falcatus e B. plicatilis ocorreram em locais com níveis elevados de fósforo e de condutividade elétrica. Em particular para B. plicatilis, maior abundância (116 org. $/ \mathrm{m}^{3}$ ) foi verificada na região central do reservatório Salto Grande, considerado supereutrófico. Essas constatações corroboram com outras pesquisas que apontam Brachionus como indicador de locais eutrofizados (ELER et al., 2003; Perbiche-Neves et al., 2013). Além disso, foi observado maior representatividade em termos de riqueza para o gênero supracitado.

Cabe ressaltar que alguns rotíferos como Filinia sp., Keratella quadrata e Trichocerca sp. também têm preferência por locais eutrofizados (SLADECEK, 1983; SouZA et al., 2013; AraúJo \& NogueIra, 2016). Com exceção de Trichocerca sp., os demais foram encontrados em abundância na represa Barra Bonita (supereutrófica). Nesse mesmo local, foi observado que a associação Conochilus unicornis/Keratella cochlearis caracterizam ambientes mais eutrofizados e a associação Polyarthra vulgaris/Keratella tropica, os menos eutrofizados (MATsumURA-TUNDISI et al., 1990).

$\mathrm{Na}$ represa de Furnas, Minas Gerais (Souza et al., 2013) e no braço Rio Grande (presente estudo), foi evidenciada uma relação entre temperatura e abundância de rotíferos. A variação da temperatura pode influenciar a solubilidade de substâncias no meio, restrigindo ou não o desenvolvimento de espécies zooplanctônicas (ZAGATTO \& Bertoletti, 2006). As taxas de ingestão de partículas por rotíferos são afetadas pela concentração de alimentos e principalmente pela temperatura. Desse modo, esse fator também influencia a produção secundária de populações do zooplâncton (GALKOVSKAJA, 1987).

Apesar da elevada abundância registrada para Asplanchna sp. (77.886 org. $\left./ \mathrm{m}^{3}\right)$, principalmente nos reservatórios de Barra Bonita (supereutrófico) e Igaratá (mesotrófico), não foi possível estabelecer uma relação entre o rotífero e os parâmetros limnológicos. Esse organismo possui hábito alimentar oportunista e a sua posição trófica está relacionada à estrutura da comunidade (KAPPES et al., 2000). Em experimentos de laboratório, verificou-se o comportamento predatório sobre diferentes espécies zooplanctônicas (CRISPIM \& BoAVIDA, 2001; NANDINI et al., 2003).

Kellicottia bostoniensis foi considerado um dos táxons mais frequentes nas amostras. Esse rotífero é nativo da América do Norte e possui ampla distribuição geográfica no Brasil e em outros países (Bomfim et al., 2016; De-CARLI et al., 2017b); é típico de ambientes eutrofizados e ricos em matéria orgânica. Em águas poluídas, as partículas suspensas sólidas e colóides derivadas das bactérias decompositoras de material orgânico, servem de alimento para espécies como K. bostoniensis, a qual pode filtrar pequenas partículas de até $10 \mu \mathrm{m}$ (LANDA et al., 2002; LuCinda et al., 2004; SeRAFiMJúNIOR et al., 2010). Registrou-se maior abundância (5.379 org. $/ \mathrm{m}^{3}$ ) de $K$. bostoniensis na entrada de água da represa Salto Grande, considerado um local hipereutrófico.

Em geral, Hexartrha sp. é encontrado em ambientes oligo-mesotróficos (ALLAN, 1976). Em dois reservatórios do estado do Paraná, Perbiche-Neves et al. (2013) constataram que a abundância esteve relacionada positivamente com os níveis de transparência da água. Por outro lado, em alguns estudos esse táxon foi mais frequente em águas eutrofizadas com alta turbidez e material em suspensão (DUGGAN et al., 2001; Sousa et al., 2008). No presente estudo, a abundância desse rotífero esteve relacionado principalmente ao estado trófico e à concentração de material em suspensão, sobretudo na entrada de água da represa Salto Grande.

Os nauplius e copepoditos de Cyclopoida apresentaram elevada densidade, principalmente na barragem do reservatório Rio Grande (6.693 org. $\left./ \mathrm{m}^{3}\right)$. Essa alta abundância de estágios iniciais é uma estratégia adaptativa para compensar a alta mortalidade antes de alcançarem a fase adulta (EspínDOLA et al., 2000). Os crustáceos ciclopóides podem se adaptar a diferentes ambientes e possuem amplo espectro alimentar (LANDA et al., 2007, Perbiche-Neves et al., 2007). O grupo mencionado é considerado uma ferramenta auxiliar no monitoramento do estado trófico de ambientes aquáticos (LANDA et al., 2007; SiLVA, 2011; Perbiche-Neves et al., 2016). No presente estudo, constatouse que o ciclopóide Mesocylops ogunnus esteve associado a locais eutróficos, como no reservatório Salto Grande.

Os crustáceos Calanoida ocorreram em locais eutróficos e com alta concentração de material em suspensão, principalmente nos reservatórios Salto Grande e Barra Bonita. $\mathrm{Na}$ região central da represa de Barra Bonita, os nauplius $\left(1.425 \mathrm{org} . \mathrm{m}^{3}\right)$ e Notodiaptomus $\mathrm{sp} .\left(1.202 \mathrm{org} / \mathrm{m}^{3}\right)$ foram considerados os mais abundantes. Por outro lado, alguns autores sugerem que os calanóides são organismos mais sensíveis às mudanças de condições ambientais (MeIRINHo \& PompÊo, 2015). Em reservatórios de São Paulo, MatsumuraTUNDISI \& TUNDISI (2003) verificaram a substituição de Argyrodiaptomus furcatus, presente em águas com baixa condutividade, por Notodiaptomus cearensis que ocorre em 
águas mais ácidas com alta condutividade. Esses organismos têm a capacidade de quebrar os filamentos de cianobactérias em pequenas partículas para sua alimentação (Bouvy et al., 2001). No caso do copépode Notodiaptomus iheringi, os indivíduos podem se alimentar e sobreviver com uma dieta restrita à cianobactéria Microcystis sp., encontrada geralmente em ambientes eutrofizados (GER et al., 2016).

Alguns cladóceros são considerados elementos-chave nos ecossistemas aquáticos por alimentarem-se basicamente de fitoplâncton, contribuindo no aumento da transparência (CARDoso et al., 2008). Em relação à essa ordem, Bosminopsis deitersi e Ceriodaphnia cornuta ocorreram em águas com maior transparência, como nas estações situadas nas represas Atibainha e Igaratá (mesotróficos). Em represas do sul do Brasil, $B$. deitersi apresentou sensibilidade às mudanças ambientais, sendo considerado indicador de baixo nível de poluição (Pedrozo \& Rocha, 2005). Por outro lado, a alta abundância de Bosmina sp. (2.036 org./ $\mathrm{m}^{3}$ ) no reservatório Salto Grande pode ser considerado um indicativo de eutrofização, corroborado por diversos estudos (Pinto-Coelho et al., 2005a,b; Sendacz et al., 2006). Quanto ao quidorídeo Alona sp., o mesmo esteve associado à condutividade elétrica e nutrientes, similar ao encontrado na represa de Furnas (SouzA et al., 2013).

Com base nos resultados apresentados, pode-se concluir que a maioria dos reservatórios estão eutrofizados. Mudanças na abundância e riqueza do zooplâncton não foram constatadas e por isso aceita-se a hipótese de que a composição de espécies não variou segundo o gradiente de impactos. Espécies como Trichocerca longiseta, Hexarthra sp., Brachiounus spp., Keratella spp., Notodiaptomus spp., Mesocyclops sp., Metacyclops sp. e Diaphanossoma spinolusum foram menos sensíveis à eutrofização. Por outro lado, os cládoceros $C$. cornuta e $B$. deitersi podem ser considerados indicadores de melhores condições de qualidade da água. Esse estudo forneceu informações relevantes sobre a qualidade de reservatórios do Estado de São Paulo. Ressalta-se a importância da caracterização físico-química e da integração das comunidades biológicas para o diagnóstico ambiental.

\section{REFERÊNCIAS BIBLIOGRÁFICAS}

Allan, J. D. 1976. Life history patterns in zooplankton. The American Naturalist 971(110):165-180.

Almeida, V. L. D. S.; LarrazÁbal, M. E. L.; Moura, A. M. \& Júnior, M. M. 2006. Rotifera das zonas limnética e litorânea do reservatório de Tapacurá, Pernambuco, Brasil. Iheringia, Série Zoologia 96(4):445-451.

ARAúJo, A. P. \& NogueIRA, E. M. DE S. 2016. Zooplâncton como bioindicador das águas do reservatório natural do povoado Olhos d'água do Souza, Glória, Bahia, Brasil. Revista Ouricuri 6(2):1116.

Barbosa, P. M. M.; Brito, S. \& Rietzler, A. C. 2006. Diversidade do zooplâncton de Minas Gerais. Revista Ciência Hoje 38(227):67-69.

Becker, V.; CAPUto, L.; OrdóÑEz, J.; Marcé, R.; Armengol, J.; Crossetti, L. O. \& Huszar, V. L. M. 2010. Driving factors of the phytoplankton functional groups in a deep Mediterranean reservoir. Water Research 44(11):3345-3354.

Bomfim, F. F.; Mantovano, T.; Schwind, L. T. F.; Palazzo F.; Bonecker C. C. \& LANSAC-TÔHA, F. A. 2016. Geographical spread of the invasive species
Kellicottia longispina (Kellicott, 1879) and K. bostoniensis (Rousselet, 1908): A scientometric approach. Acta Scientiarium, Biological Sciences 38(1):29-36.

Bouvy, M.; Pagano, M. \& Troussellier, M. 2001. Effects of a cyanobacterial bloom (Cylindrospermopsis raciborskii) on bacteria and zooplankton communities in Ingazeira reservoir (northeast Brazil). Aquatic Microbial Ecology 25:215-227.

Campos, E. V. R.; Oliveira, J. L. \& Fraceto, L. F. 2014. Applications of controlled release systems for fungicides, herbicides, acaricides, nutrients, and plant growth hormones: a review. Advanced Science, Engineering and Medicine 6:1-15.

Cardoso, L. S.; Ramos, J. D. \& Mello, H. O. DE O. 2008. Composição, densidade e abundância das populações de Cladocera, Copepoda e Rotifera de Áreas de Proteção Permanente do rio Uberabinha. Em Extensão 7(2):95-106.

Castilho, M. C. A.; Wisniewski, M. J. S, Wisniewski, C. \& Silva, E. Dos S. 2016. Quantifying zooplankton species: use of richness estimators. Iheringia, Série Zoologia 106(1):e2016011.

CETESB. 2012. Norma Técnica L5.304 - Zooplâncton de Água Doce. Métodos Qualitativo e Quantitativo (Método de ensaio). São Paulo, CETESB. 16p.

CrisPim, M. C. \& BOAVIDA, M. J. 2001. Impacto da predação por peixes e copépodes na comunidade zooplanctônica do reservatório do Maranhão (Portugal). Revista Nordestina de Biologia 15(2):49-67.

Cole, G. A. 1979. Textbook of Limnology. 2ed. Saint Louis, Mosby Company. $425 \mathrm{p}$.

Cunha, D. G. F.; Calijuri, M. C. \& Lamparelli, M. C. 2013. A trophic state index for tropical/subtropical reservoirs (TSItsr). Ecological Engineering 60:126-134.

DAJOZ, R. 1983. Ecologia geral. Petrópolis, Vozes. 472p.

DANTAS-SILVA, L. T. \& DANTAS, E. W. 2013. Zooplâncton e a eutrofização em reservatórios do Nordeste brasileiro. Oecologia Australis 17(2):53-58.

De-Carli, B. P.; Lopez-Doval, J. C.; Rodrigues, E. H. C. \& Pompêo, M. L. M. 2017a. Variação espacial e sazonal do zooplâncton nos reservatórios do Sistema Cantareira, Brasil. Revista Ambiente e Água 12(4):666-679.

De-Carli, B. P; Albuquerque, F. P. de; Bayanov, N. G.; Moschini-Carlos, V. \& PoMPÊO, M. L. M. 2017b. Dispersão e primeiro registro da espécie invasora Kellicottia bostoniensis (Rotifera: Brachionidae) em dois reservatórios brasileiros. Oecologia Australis 21(4):455-460.

Duggan, I. C.; Green, J. D. \& Shiel, R. J. 2001. Distribution of rotifers in North Island, New Zealand, and their potential use as bioindicators of lake trophic state. Hydrobiologia 446/447:155-164.

EC - European Commission. 2012. Report from the Commission to the European Parlament and the Council on the Implementation of the Water Framework Directive (2000/ 60/EC): River Basin Management Plans. Brussels, European Comission. 15p.

Eler, M. N.; Pareschi, D. C.; Espíndola, E. L. G. \& Barbosa, D. S. 2003. Ocorrência de Rotifera e sua relação com o estado trófico da água em pesque-pague na bacia do rio Mogi-Guaçu - SP. Boletim Técnico do CEPTA 16:41-56.

ElmoOR-LOUREIRO, L. M. A. 1997. Manual de identificação de cladóceros límnicos do Brasil. Brasília, Editora Universa, Universidade Católica de Brasília. 156p.

Espíndola, E. L. G.; Matsumura-Tundisi, T.; Rietzler, A. C. \& Tundisi, J. G. 2000. Spatial heterogeneity of the Tucuruí reservoir (State of Pará, Amazonia, Brazil) and the distribution of zooplancton species. Revista Brasileira de Biologia 60(2):179-193.

Freitas, G. T. DE P.; Crispim, M. C. \& Júnior, H. N. M. 2012. Effects of net cages on the vertical distribution of zooplankton in a semi-arid reservoir, northeastern Brazil. Acta Limnologica Brasiliensia 24(2):140-148.

GALKOVSKAJA, G. A. 1987. Planktonic rotifers and temperature. Hydrobiologia 147(1):307-317.

Gazonato-Neto, A. J.; Silva, L. C.; Saggio, A. A. \& Rocha, O. 2014. Zooplankton communities as eutrophication bioindicators in tropical reservoirs. Biota Neotropica 14(4):1-12.

Ger, K. A.; Leitão, E. \& Panosso, E. 2016. Potential mechanisms for the tropical copepod Notodiaptomus to tolerate Microcystis toxicity. Journal of Plankton Research 38(4):843-854.

Kappes, H.; Mechenich, C. \& Sisch, U. 2000. Long-term dynamics of Asplanchna priodonta in Lake Windsborn with comments on the diet. Hydrobiologia 432:91-100. 
Kimmel, B. L.; Lind, O. T. \& Paulson, L. J. 1990. Reservoir primary production. In: Thorton, K. W.; Kimmel, B. L. \& PAYNe, F. E. eds. Reservoir limnology: ecological perspectives. Toronto, John Wiley, Wiley-Interscience, p. 133-194.

KorolefF, F. 1976. Determination of nutrients. In: GrasSHOFF, K. ed. Methods of seawater analysis. Weinheim, Wiley-VCH Verlag, p. 117-181.

KosTe, W. 1978. Rotatoria, die Rädertiere Mitteleuropas: Überordnung Monogononta: ein Bestimmungswerk. Berlin, Gebrüder Borntraeger. $673 p$.

Kuhlmann, M. L.; Johnscher-Fornasaro, G.; Ogura, L. L. \& Imbimbo, H. R. V. 2012. Protocolo para o biomonitoramento com as comunidades bentônicas de rios e reservatórios do estado de São Paulo. São Paulo, CETESB. Disponível $\mathrm{em}<\mathrm{http}$ ://laboratorios.cetesb.sp.gov.br/wp-content/ uploads/sites/47/2015/01/protocolo-biomonitoramento-2012.pdf $>$. Acesso em 18/11/2016

Landa, G. G.; Barbosa, F. A. R.; Rietzler, A. C. \& Maia-Barbosa, P. M. 2007. Thermocyclops decipiens (Kiefer, 1929) (Copepoda: Cyclopoida) as indicator of water quality in the state of Minas Gerais, Brazil. Brazilian Archives of Biology and Technology 50(4):695-705.

LANDA, G. G.; Del Aguila, L. M. R. \& CoElho, R. M. P. 2002. Distribuição espacial e temporal de Kellicottia bostoniensis (Rousselet, 1908) (Rotifera) em um grande reservatório tropical (reservatório de Furnas), estado de Minas Gerais, Brasil. Acta Scientiarium 24(2):313-319.

LEGENDRE, P. \& LEGENDRE, L. 1998. Numerical ecology. Amsterdan, Elsevier Science. 853p.

Lopez-Doval, J. C.; Montagner, C. C.; Albuquerque, A. F.; MoschiniCarlos, V.; Umbuzeiro, G. \& Pompêo, M. 2017. Nutrients, emerging pollutants and pesticides in a tropical urban reservoir: Spatial distributions and risk assessment. Science of The Total Environment 575:1307-1324.

LORENZEN, C. J. 1967. Determination of chlorophyll and pheo-pigments: spectrophotometric equations. Limnology Oceanography 12(2):343-346.

Loureiro, B. R.; Costa, S. M.; Macedo, C. F.; Huszar, L. M. \& Branco, C. W. C. 2011. Comunidades zooplanctônicas em sistemas de criação de peixes. Boletim Instituto de Pesca de São Paulo 37(1):47-60.

Lucinda, I.; Moreno, I. H.; Melão, M. G. G. \& Matsumura-Tundisi, T. 2004. Rotifers in freshwater habitats in the Upper Tietê River Basin, São Paulo State, Brazil. Acta Limnologica Brasiliensia 16(3):203-224.

Mackereth, F. J. H.; Heron, J. \& TAlling, J. F. 1978. Water analysis: some revised methods for limnologists. Dorset, Freshwater Biology Assessment. 121p.

Matsumura-Tundisi, T. \& Tundisi, J. G. 1976. Plankton studies in a lacustrine environment. I. Preliminary data on zooplankton ecology of Broa Reservoir. Oecologia 25:265-270.

Matsumura-Tundisi, T.; Leitão, S. N.; Aguena, L. S. \& Miyahara, J. 1990 Eutrofização da represa de Barra Bonita: estrutura e organização da comunidade de Rotifera. Revista Brasileira de Biologia 50(4):923-935.

Matsumura-Tundisi, T. \& Tundisi, J. G. 2003. Calanoida (Copepoda) species composition changes in the reservoirs of São Paulo State (Brazil) in the last twenty years. Hydrobiologia 504:215-222.

Matsumura-TundisI, T. \& TundisI, J. G. 2005. Plankton richness in a eutrophic reservoir (Barra Bonita Reservoir, SP, Brazil). Hydrobiologia 542:367-378.

Matsumura-Tundisi, T.; Rietzler, A. C. \& Tundisi, J. G. 1989. Biomass (dry weight and carbon content) of plankton Crustacea from Broa Reservoir (São Carlos, SP, Brazil) and its fluctuation across one year. Hydrobiologia 179:229-236.

MEIRINHO, P. A. \& Pompêo, M. 2015. Histórico de estudos sobre a comunidade zooplanctônica do reservatório Rio Grande ao longo do tempo e sua heterogeneidade espacial. In: POMPÊO, M.; MosChINI-CARLos, V.; Nishimura, P.; Cardoso-Silva, S. \& Lopez-Doval, J. C. eds. Ecologia de reservatórios e interfaces. São Paulo, Instituto de Biociências da Universidade de São Paulo. 460p.

Milligan, G. W. \& CoOper, M. C. 1988. A Study of Standardization of Variables in Cluster Analysis. Journal of Classification 5:181-204.

Nandini, S.; Perez-Chavez, R. \& Sarma, S. S. 2003. The effect of prey morphology on the feeding behaviour and population growth of the predatory rotifer Asplanchna sieboldi: a case study using five species of Brachionus (Rotifera). Freshwater Biology 48:2131-2140.

Navarro, E.; Bacardit, M.; Caputo, L.; Palau, T. \& Armengol, J. 2006. Limnological characterization and flow patterns of a three-coupled reservoir system and their influence on Dreissena polymorpha populations and settlement during the stratification period. Lake Reservoir Management 22(4):293-302.

Nogrady, T. \& Segers, H. 2002. Rotifera: Asplanchnidae, Gastropodidae, Lindiidae, Microcodidae, Synchaetidae, Trochosphaeridae and Filinia. In: Dumont, H. J. ed. Guides to the Identification of the microinvertebrates of the continental waters of the world. Netherlands, SPB Academic Publishers, 6. 264p.

NORDI, N. \& WATANABE, T. 1978. Nota preliminar sobre rotíferos (zooplâncton) do Açude Epitácio Pessoa, Boqueirão, Paraíba. Revista Nordestina de Biologia 1(1):31-39.

PÁDUA, J. coord. 2009. Manual para a avaliação da qualidade biológica da água em lagos e albufeiras segundo a DQA - Protocolo de amostragem e análise para o fitoplâncton. Lisboa, INAG, I. P. 67p.

Pedrozo, C. DA S. \& Rocha, O. 2005. Zooplankton and water quality of lakes of the Northern Coast of Rio Grande do Sul State, Brazil. Acta Limnologica Brasiliensia 17(4):445-464.

Perbiche-Neves, G.; Serafim JR., M.; Ghidini, A. R. \& Brito, L. 2007. Spatial and temporal distribution of Copepoda (Cyclopoida and Calanoida) of an eutrophic reservoir in the basin of upper Iguaçu River, Paraná, Brazil. Acta Limnologica Brasiliensia 19(4):393-406.

Perbiche-Neves, G.; Boxshall, G. A.; Previattelli, D.; Nogueira, M. G.; Rосна, C. E. F. 2015. Identification guide to some Diaptomid species (Crustacea, Copepoda, Calanoida, Diaptomidae) of "de la Plata" River Basin (South America). Zookeys 497:1-111.

Perbiche-Neves, G.; Fileto, C.; Laço-Portinho, J.; Troguer, A. \& SerafimJÚNIOR, M. 2013. Relations among planktonic rotifers, cyclopoid copepods, and water quality in two Brazilian reservoirs. Latin American Journal of Aquatic Research 41(1):138-149.

Perbiche-Neves, G.; Saito, V. C.; Previattelli, D.; Da Rocha, C. E. F. \& Nogueira, M. G. 2016. Cyclopoid copepods as bioindicators of eutrophication in reservoirs: Do patterns hold for large spatial extents? Ecological Indicators 70:340-347.

Pinto-Coelho, R.; Pinel-Alloul, B.; Méthot, G. \& Havens, K. E. 2005 a. Crustacean zooplankton in lakes and reservoirs of temperate and tropical regions: variation with trophic status. Canadian Journal of Fisheries and Aquatic Sciences 62:348-361.

Pinto-Coelho, R. M.; Bezerra-Neto, J. F. \& Morais-JR., C. A. I. 2005b. Effects of eutrophication on size and biomass of crustacean zooplankton in a tropical reservoir. Brazilian Journal of Biology 65(2):325-338.

Santos, T. G. Dos; Gusmão, L. M. O.; Neumann-Leitão, S. \& Cunha, A. G. DA. 2009. Zooplâncton como indicador biológico da qualidade ambiental nos estuários dos rios Carrapicho e Botafogo, Itamaracá-PE. Revista Brasileira Engenharia de Pesca 4(1):44-56.

SendacZ, S.; CalefFi, S. \& SANTos-SoARES, J. 2006. Zooplankton biomass of reservoirs in different trophic conditions in the State of São Paulo, Brazil. Brazilian Journal of Biology 66(1):337-350.

Sendacz, S.; Kubo, E. \& Cestarolli, M. A. 1985. Limnologia de reservatórios do sudeste do Estado de São Paulo, Brasil. VIII. Zooplâncton. Boletim do Instituto de Pesca 12(1):187-207.

Serafim-Júnior, M.; Perbiche-Neves, G.; de Brito, L.; GhidinI, A. R. \& Casanova, S. M. C. 2010. Variação espaço-temporal de Rotifera em um reservatório eutrofizado no sul do Brasil. Iheringia, Série Zoologia 100(3):233-241

SILVA, W. M. 2008. Diversity and distribution of the free-living freshwater Cyclopoida (Copepoda: Crustacea) in the Neotropics, Brazil. Brazilian Journal of Biology 68:1099-1106.

SiLVA, W. M. 2011. Potential use of Cyclopoida (Crustacea, Copepoda) as trophic state indicators in tropical reservoirs. Oecologia Australis 15(3):511-521.

SlaDECEK, V. 1983. Rotifers as indicators of water quality. Hydrobiologia 100:169-201.

Sousa, W.; AtTayde, J. L.; Rocha, E. S. \& Eskinazi, S. E. M. 2008. The response of zooplankton assemblages to variations in lakes in semi-arid northeastern Brazil. Journal of Plankton Research 30(6):699-708.

SouZA, T. R.; NunEs, M. V. \& DA SILVA, L. C. 2013. Comunidade zooplanctônica e seu uso como bioindicadora do estado trófico de uma região do reservatório da UHE de Furnas. IX Fórum Ambiental da Alta Paulista 9(11):546-559. 
Teixeira, C.; Tundisi, J. G. \& Kutner, M. B. 1965. Plankton studies in a mangrove II. The standing stock and some ecological factors. Boletim Instituto Oceanográfico 24:23-41.

Tundisi, J. G. 1994. Tropical South America: present and perspectives. In: MARGALEF, R. Limnology now: a paradigm of planetary problems. Amsterdam, Elsevier Science, p. 353-424.

Tundisi, J. G. 2008. Recursos hídricos no futuro: problemas e soluções. Estudos Avançados 22(63):7-16.

TundisI, J. G. 2014. Recursos hídricos no Brasil: problemas, desafios e estratégias para o futuro. Rio de Janeiro, Academia Brasileira de Ciências. $76 \mathrm{p}$.

Tundisi, J. G. \& Matsumura-Tundisi, T. 2008. Limnologia. São Paulo, Oficina de Textos. 632p.

Vad, C. S.; Horvath, Z.; Kiss, K. T.; Toth, B.; Pentek, A. L. \& Acs, E. 2013. Vertical distribution of zooplankton in a shallow peatland pond: the limiting role of dissolved oxygen. Annales de Limnologie - International Journal of Limnology 49:275-283.

VAN DEN Brink, P. J. \& Ter BraAK, C. J. F. 1998. Multivariate analysis of stress in experimental ecosystems by Principal Response Curves and similarity analysis. Aquatic Ecology 32(2):163-178.

VAlderramA, J. C. 1981. The simultaneous analysis of total nitrogen and total phosphorus in natural waters. Marine Chemistry 10:109-222.

Wetzel, R. G. \& Likens, G. E. 1991. Limnological Analyses. New York, Springer-Verlag. 391p.

Zaganini, R. L.; Perbiche-Neves, G.; Naliato, D. A. O. \& Carvalho, E. D. 2011. Baixa diversidade de zooplâncton na desembocadura de uma represa eutrófica (SP, Brasil): reflexo da poluição? Estudos de Biologia 32/33:76-81.

Zagatto, P. A. \& Bertoletti, E. 2006. Ecotoxicologia aquática - Princípios e Aplicações. São Carlos, RiMa. 478p.

ZAR, J. H. 2010. Biostatistical Analysis. 5ed. New Jersey, Prentice Hall. 944p. 